\title{
Performance Measurements Towards Improved Manufacturing Vehicle Safety
}

\author{
Roger Bostelman \\ NIST \\ 100 Bureau Drive, Stop 8230 \\ Gaithersburg, MD 20899 \\ (301) 975-3426 \\ roger.bostelman@nist.gov
}

\author{
Will Shackleford \\ NIST \\ 100 Bureau Drive, Stop 8230 \\ Gaithersburg, MD 20899 \\ (301) 975-4286 \\ shackle@nist.gov
}

\begin{abstract}
In this paper, we describe the current 2D (two dimensional) sensor used for industrial vehicles and ideal sensor configurations for mounting 3D imagers on manufacturing vehicles in an attempt to make them safer. In a search for the ideal sensor configuration, three experiments were performed using an advanced 3D imager and a color camera. The experiments were intended to be useful to the standards community and manned and unmanned forklift and automated guided vehicle industries. The imager that was used was a 3D Flash LIDAR (Light Detection and Ranging) camera with $7 \mathrm{~m}$ range and rapid detection. It was selected because it shows promise for use on forklifts and other industrial vehicles. Experiments included: 1) detection of standard sized obstacles, 2) detection of obstacles while highly reflective surfaces are also within detection range, and 3) detection of forklift tines above the floor. We briefly describe these experiments and reference their detailed reports.
\end{abstract}

\section{Categories and Subject Descriptors}

I2.10 [Vision and Scene Understanding]: 3D/stereo scene analysis

\section{General Terms}

Performance, Design, Experimentation, Standardization

\section{Keywords}

3D Flash LIDAR, Forklifts, Powered Industrial Trucks, Automated Guided Vehicles (AGV), ANSI/ITSDF B56.5

\section{INTRODUCTION}

The National Institute of Standards and Technology (NIST), Intelligent Systems Division (ISD) has been performing measurements to be used as background information for advancing standards and for the manned and unmanned vehicle and sensors industries in an attempt to make forklifts and other vehicles safer. The Occupational Safety and Health Administration states: [1] "Each year, tens of thousands of injuries related to powered industrial trucks (PIT), or forklifts,

This paper is authored by employees of the United States Government and is in the public domain.

PerMIS'09, September 21-23, 2009, Gaithersburg, MD, USA. ACM 978-1-60558-747-9/09/09 occur in US workplaces... Most incidents also involve property damage, including damage to overhead sprinklers, racking, pipes, walls, and machinery. Unfortunately, most employee injuries and property damage can be attributed to lack of safe operating procedures, lack of safety-rule enforcement, and insufficient or inadequate training." The statement suggests the need for improving driver's knowledge, although safer vehicles can also help. Obstacle detection sensing that completely surrounds the vehicle could augment the driver's or autonomous vehicle's environmental awareness. Driver alerts and/or autonomous slow or stop vehicle operations are then possible based on this sensor information and, therefore, could provide safer vehicles.

NIST ISD has been working for several years with the Industrial Truck Standards Development Foundation (ITSDF) which manages "ITSDF B56.5 Safety Standard for Guided Industrial Vehicles and Automated Functions Of Manned Industrial Vehicles" [2] as approved by the American National Standards Institute (ANSI). NIST's involvement with the B56.5 standard includes performance measurements of advanced non-contact sensors for automated guided vehicles (AGV's), and has led to proposed changes to the standard. AGVs are typically programmed to follow prescribed paths but still need sensors to detect obstacles such as closed doors, equipment, personnel or material left temporarily in the vehicles' paths. Currently they rely heavily on 2D line scanners, while some are also equipped with a physical bumper as the final backup to stop the vehicle. The 2D line scanners work well with ground-based vertical obstacles but it takes many sensors to completely protect against overhanging obstacles and even then they do not scan the full volume of space the vehicle travels through. Figure 1 shows how 2D line scanning sensors are typically oriented on vehicles to aid detection of overhanging obstacles. The lower triangular region near the vehicle would be undetected at vehicle startup. Side and top sensors in the figure could themselves become obstacles if mounted as shown. The red dotted lines depict sensor scan-lines. From the side view, it is clear that 2D sensors of this type may not detect obstacles that are not within the sensor scan-line or have already passed through the scan-line. As shown, the scanner can miss an overhanging obstacle completely when directly in front of the sensor housing or if the obstacle is detected it may be too late to stop or slow the vehicle. 


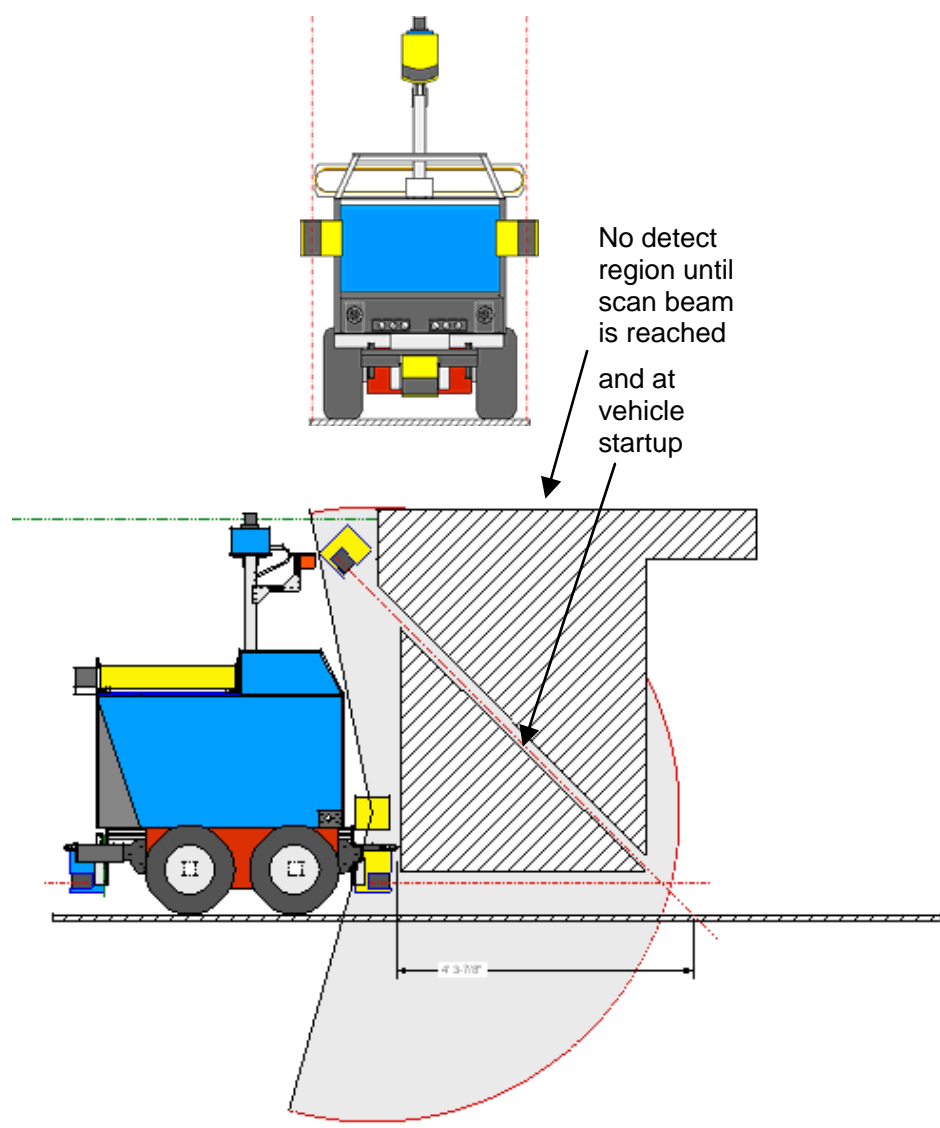

Figure 1 - Graphic showing front (top) and side (bottom) views of typical 2D line scanning LIDAR sensors mounting configuration on AGV's.

3D Flash LIDAR technology has led to a relatively new class of range imaging sensors with the potential to scan $3 \mathrm{D}$ volumes faster than scanning LIDAR systems. Capabilities of this type of sensor could dramatically change the way sensors are used on manufacturing vehicles. This concept will be further explained in Section 2.

To evaluate this class of sensors, a consortium of AGV vendors was formed that took preliminary data with several Flash range imaging systems and selected one for further development and investigation. This is the sensor used for this work. The data collection system was integrated with a NIST-developed vehicle control system, the Mobility Open Architecture Simulation and Tools (MOAST) framework. [3] This allowed the system to collect data while the vehicle was driving autonomously.

Three experiments were completed providing background data towards proving the usefulness of advanced 3D Flash LIDAR cameras on forklifts and PITs. Experiments included: 1) detection of obstacles while highly reflective surfaces are also within the camera's field of view, 2) detection of forklift tines above the floor, and 3) detection of obstacles specified in the ITDSF/ANSI standard. Each of these experiments is briefly explained in Section 3.

\section{IDEAL 3D VEHICLE SENSING CONCEPT}

Ideally, the volume that completely surrounds the manned or unmanned vehicle should be sensed to ensure a safe manufacturing environment. Ideal 3D vehicle sensing volume concepts are depicted in the graphics shown in Figures 2 and 3. Figure 2 shows top and front views of the ideal 3D sensing volumes for an AGV in green completely surrounding the vehicle, extending beyond the vehicle to include a safe stopping distance. Figure 3 depicts the concept of using multiple, 3D imaging sensors to measure the volume surrounding an AGV (Figure 3, top) and a forklift (Figure 3, bottom). The orange triangles represent sensor fields of view (FOV). The bottom graphic shows a forklift carrying a double-height load and a movable arm (green) that carries a 3D imager to look over and in front of the load. Also, especially for high reach forklifts, a sensor is needed on the mast top to sense overhead obstacles such as ceilings.

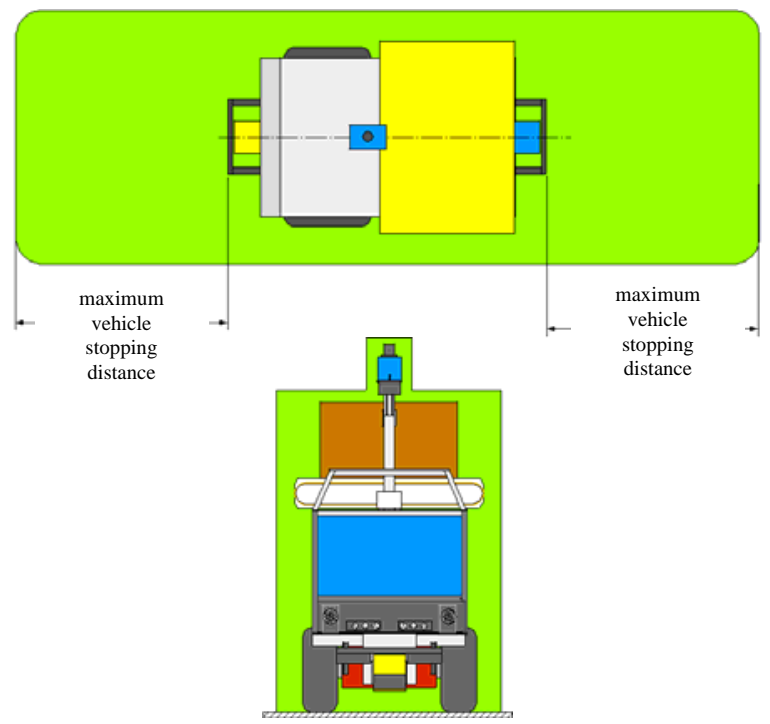

Figure 2 - Top view (top) and front view (bottom) of the ideal 3D sensing volume for AGVs.

\section{3D IMAGING EXPERIMENTS}

As a preliminary to the implementation of the $3 \mathrm{D}$ sensing scenarios posed in the previous section, performance measurements are required of advanced 3D imagers. 3D Flash LIDAR, a time-of-flight range measurement sensor, is still fairly new to the vehicle industry and requires further experimentation in real or simulated manufacturing environments to ensure safe vehicle operations. The sensor used for these experiments measures non-ambiguity range to $7.5 \mathrm{~m}$ for each of its $176 \mathrm{x} 144$ pixels with an internal modulating frequency of $20 \mathrm{MHz}$. The following subsections briefly discuss 3D Flash LIDAR experiments performed at NIST for use as safety sensors on manufacturing vehicles.

\subsection{Detection of Standard Obstacles}

NIST has recently performed measurements with results [4] to be used as background information towards changes to the ITSDF B56.5 Safety Standard with regard to non-contact sensors detecting standard test pieces. The B56.5 standard defines safety requirements relating to the elements of design, 
operation, and maintenance of powered, not mechanically restrained, unmanned automatic guided industrial vehicles and automated functions of manned industrial vehicles. Optical and acoustic sensors were tested in these experiments on B56.5 standard test piece sizes, as well as a large flat metal plate, cinder block, and other test pieces and test piece coverings. Over 120 data sets from 21 different tests using a variety of test piece configurations, coverings and layouts were performed in a NIST laboratory. For this paper, our focus is only on the optical, 3D Flash LIDAR sensor experiments and results.

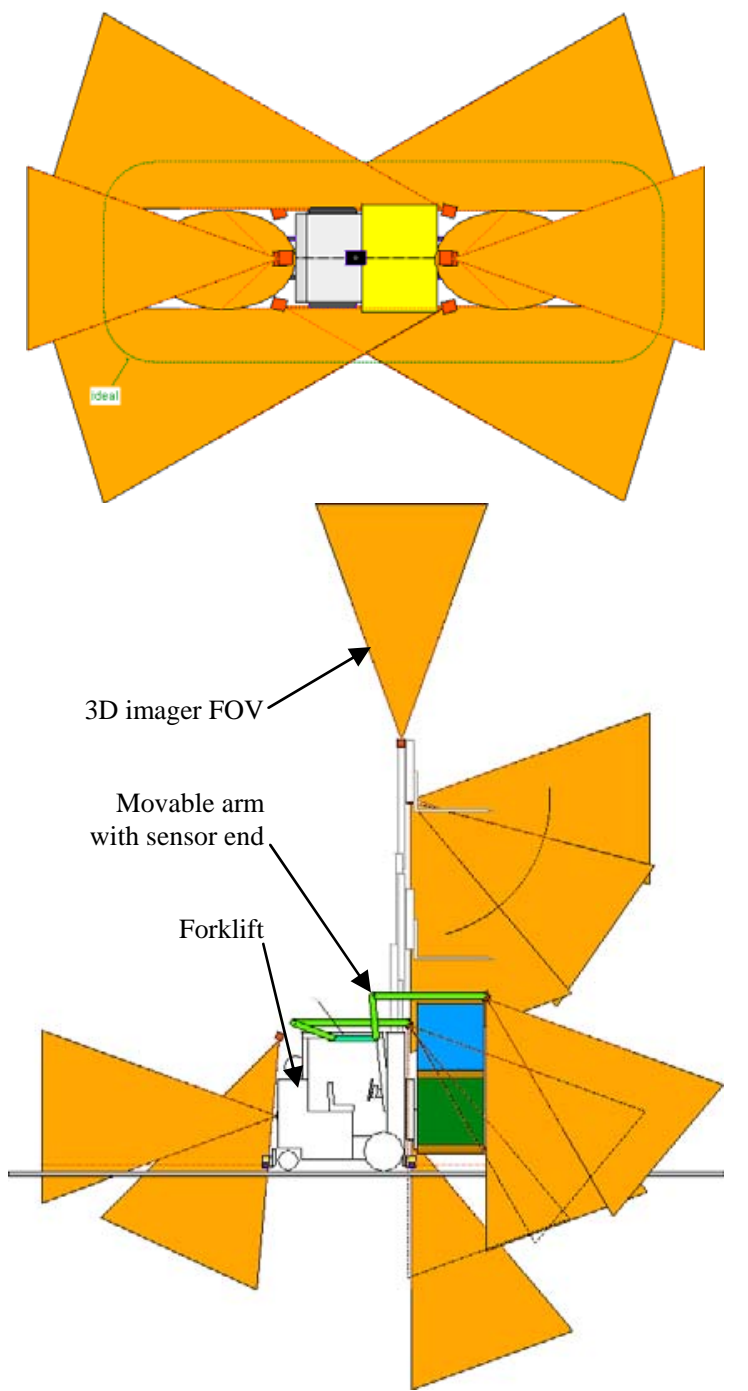

Figure 3 - (top) Top view of an AGV with multiple 3D imaging sensors surrounding the vehicle and measuring to and beyond the ideal sensing region, (bottom) side view of a forklift with multiple 3D imagers showing sensinging volumes required to reach the ideal sensing capability. The forklift is shown carrying a double-height palletized load and the green arm carries a 3D imager to look over and in front of the load.

The ITSDF B56.5-2005 Safety Standard section on non-contact sensing devices states that if the sensor is used as the primary emergency device, the sensor shall be fail-safe in its operation and mounting and shall stop the vehicle travel prior to contact between the vehicle structure and the object detected. Test pieces are to be detected in the main direction of travel and are to be: a $600 \mathrm{~mm}$ cylinder with a $200 \mathrm{~mm}$ diameter lying at any angle to and anywhere on the path of the vehicle and a second, $400 \mathrm{~mm}$ cylinder with a $70 \mathrm{~mm}$ diameter set vertically anywhere fully within the path of the vehicle. The test pieces described in the standard are of specific size, originally based on the British EN1525 standard. [5] Because the standard is based on contact sensors, however, there are currently no restrictions on test piece coverings. Requirements are necessary because noncontact sensors may react differently to various materials to be detected. A sensor may or may not detect a particular material and a failure to detect could cause a safety hazard. An example might be that a person wearing dark clothes may not be detected by some optical sensors. Also, only cylindrical test pieces are listed in the standard and perhaps provide better performance than flat test pieces might when positioned at specific angles with respect to the sensors. The experiments were designed to evaluate these additional problems, with the goal of suggesting new language to add to the standard. Figure 4 shows the experimental setup of sensors and test pieces.

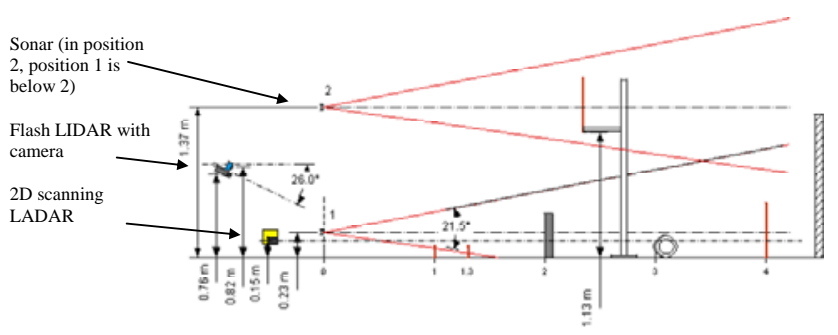

Figure 4 - Graphic of experimental test setup showing dimensions (in meters). Sensors are shown to the left and targets

shown in the figure are, from middle to right: flat targets positioned horizontally at $1 \mathrm{~m}$ and $1.3 \mathrm{~m}$, a $70 \mathrm{~mm}$ dia. $\mathrm{x} 400$ $\mathrm{mm}$ long cylinder at $2 \mathrm{~m}$, a raised flat target mounted $1.13 \mathrm{~m}$ vertically above the floor on a floor stand, a $200 \mathrm{~mm}$ dia. x 600 $\mathrm{mm}$ long cylinder lying horizontally at $3 \mathrm{~m}$, and a flat target oreintedvertically at $4 \mathrm{~m}$. A background cardboard wall is to the far right.

Various coverings over the test pieces, including cotton cloth, paint, and standard color patches, were used to evaluate how well the sensors could detect the pieces under different conditions. The coverings used were representative of clothes and colors that may be worn by people or of manufactured or other industrial materials that may be near vehicles in real world situations. Figure 5 shows a standard sized horizontal cylinder test piece with three standard reflectance paper patches with $6 \%$ (density of $1.22 \mathrm{D}$ ), $50 \%$ (density of $0.30 \mathrm{D}$ ) and white. As shown, the right range image is nearly washed out and undetected by one Flash LIDAR sensor.

Overall results from using the 3D Flash LIDAR sensor included the following points:

- The sensors used in the tests show a noticeable difference between highly reflective versus relatively low reflective targets.

- In a horizontal cylinder test, two cylinders placed side by side were difficult to detect at $2 \mathrm{~m}$ range and undetected beyond $2 \mathrm{~m}$ with the flat black painted cardboard cylinder being much more difficult to detect than the metal cylinder. The cylinder appears to blend in with the floor. The 
cylinders are detected only when they are in front of a background obstacle or wall.

- In a flat plate detection test, the $1 \mathrm{~m}, 0^{\circ}$ test produced poor results. The obstacles at this distance and angle were difficult to detect by the researcher in the range image, although they were detected in the intensity image. However, at $1 \mathrm{~m}$ and tilted at a $45^{\circ}$ angle with either the horizontal or vertical axis, the target was detected in the range and intensity data. Beyond $1 \mathrm{~m}$, all flat targets were detected except when the target was covered with reflective foil. There were no problems detecting painted or unpainted cinder blocks.

- In the flat plate glass test, the glass was never detected because the sensor saw through the glass. The frame holding the glass was detected.

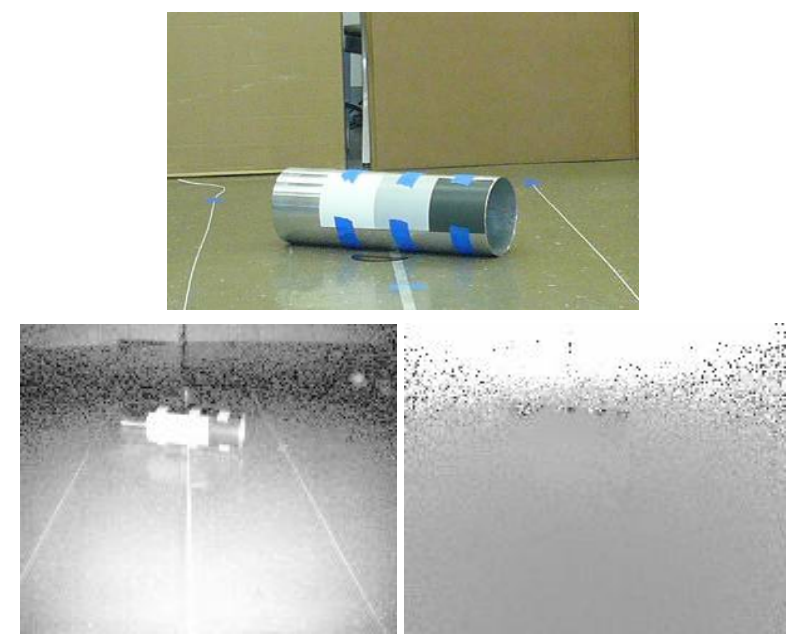

Figure 5 - (top) Close-up of the test piece covered with standard reflectance bars. (bottom) 3D Flash LIDAR intensity (left) and range (right) data from the horizontal cylinder.

\subsection{Effects of Highly Reflective or Emissive Surfaces}

Experiments were conducted to better understand how 3D Flash LIDAR reacts to highly reflective objects in their fields of view. Such objects are typical of AGV laser positioning system reflectors. Highly reflective surfaces may cause distortions in the data which could affect how the vehicle sensors perceive their surroundings, potentially causing them to miss obstacles in the vehicle path. Here we briefly explain the experiment and results. Full details can be reviewed in [6]. The Flash LIDAR sensors that were tested emit a short pulse of light at $870 \mathrm{~nm}$ into the environment and sense returned illumination within their field of view using an array of $76 \times 144$ pixels. The sensor has a FOV of $0.26 \mathrm{rad} \times 0.22 \mathrm{rad}\left(47.5^{\circ} \times 39.6^{\circ}\right)$ and a range resolution of $7.5 \mathrm{~m}$. Two experiments were completed: 1 .) highly reflective object test and 2.) sensor passing by a light source.

The 3D Flash LIDAR was fixed to the front edge of a small table on wheels at a height of approximately $1 \mathrm{~m}$ above the floor. We consulted with an AGV manufacturer to establish the typical size and mounting height at which AGV positioning reflectors (cylinders) were typically mounted. We set up a 0.75 $\mathrm{m} \times 0.1 \mathrm{~m}$ diameter reflector so that the sensor beam him the center of the reflector at $2.5 \mathrm{~m}$ above the floor., as well as at $2 \mathrm{~m}$ (called 0 height), $1.5 \mathrm{~m}$ (called $-0.5 \mathrm{~m}$ height) and $1 \mathrm{~m}$ (called $1 \mathrm{~m}$ height) above the floor and at ranges of $7 \mathrm{~m}$ to $3.5 \mathrm{~m}$ from the 3D sensor. The bottom of the reflector cylinder was placed on these surface heights. Figure 3 (left) shows a drawing of the experimental layout. Figure 3 (middle) shows a photo of the experimental set-up showing several obstacles in the sensor's FOV, including a reflector brightly illuminated by the camera's flash, and the data capture computer laptop (lower right). Figure 6 (bottom) shows the data captured from the scene using a 3D Flash LIDAR. The yellow arrows show the chairs in the left and right in the photo and range data. Note that the side view (bottom-right) of the data is skewed (vertical surfaces appear angled back) as a result of the highly reflective surface from the reflector.

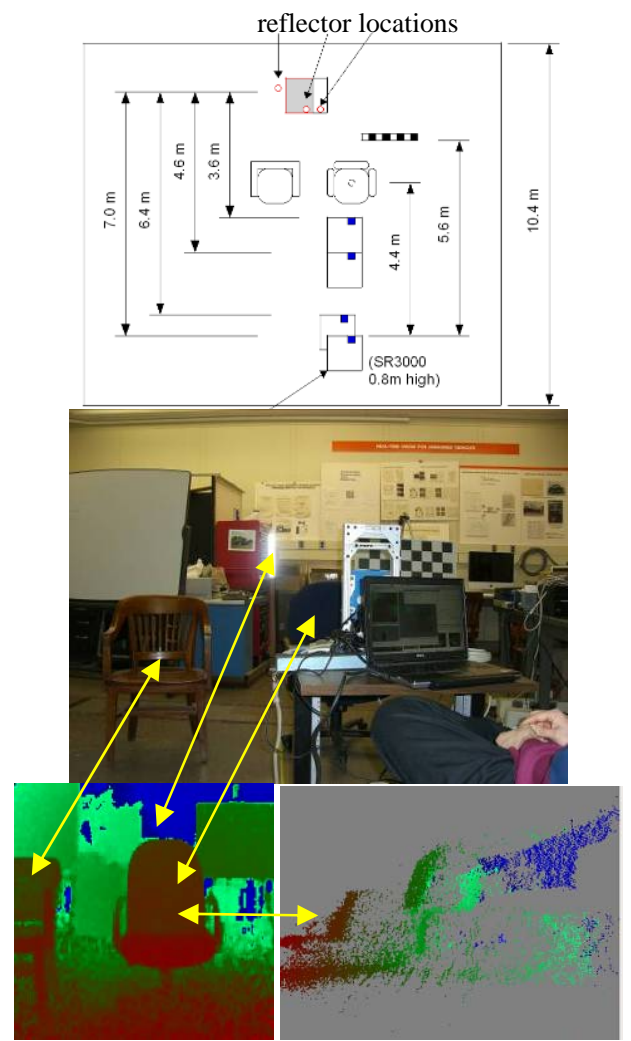

Figure 6: (top) Graphic showing the top view of the experimental setup of the highly reflective surface test; (middle) photo of the experimental set-up showing several obstacles in sensor view, a reflector illuminated by the camera's flash, and the data capture computer laptop (lower right in the photo); (bottom-left) captured data showing the front view and (bottomright) side view of the scene using a 3D Flash LIDAR sensor. The yellow arrows point to the same chairs and reflector in the photo and data.

Results show that when the 3D Flash LIDAR was mounted low so as to not capture highly reflective returns, the received data was not distorted. When the reflector was detected within the scene, the image was distorted in that region. Masking out 
upper rows of the sensor's light emitting diodes helped to remove some distortion of the scene for high mounted reflectors. Further, we determined two options are possible to alleviate the problem: (a) 3D Flash LIDAR threshold can be adjusted and/or (b) use a NIST-developed post-process intensity removal tool. For (a), the 3D Flash LIDAR can be adjusted to remove high intensity data directly from the received camera data. We added a simple software slide "adjuster" tool for simplicity. This can be run as a constant image adjuster in real time. Thresholding is performed within the camera so the host computer is not burdened by this extra task.

For (b) we developed an algorithm built into our display tool that finds and counts reflectors in the scene and masks out a variable-size region around the reflector based on the area of high intensity returns. Both the threshold and the masking algorithms can run in real-time. Only algorithm (b) can be run as image post-processing because the thresholding is carried out in the sensor. Neither algorithm corrects the distorted data. They only detect the region of the image where distortion is likely and data should not be used. Whether this is useful depends on factors outside the sensor processing system. For a particular application it may be acceptable for the AGV to run slowly enough while near a reflector to rely only on a physical bumper or other safety sensor instead of a 3D flash imager. Figure 7 shows color camera (left) and 3D Flash LIDAR intensity and range data (right) of (a) no reflector in the scene, (b) a reflector laying on the chair, (c) a reflector in the chair where the threshold algorithm has removed it from the data, (d) a reflector in the chair and the masking algorithm has removed the region of high reflectivity.

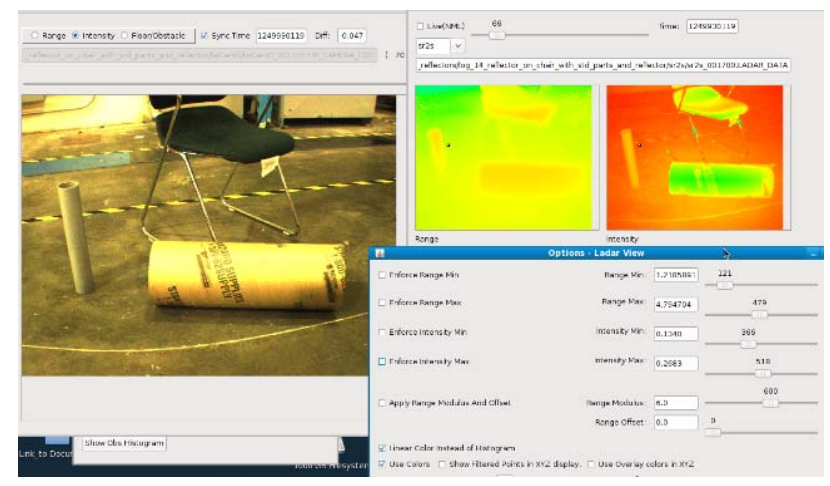

(a)

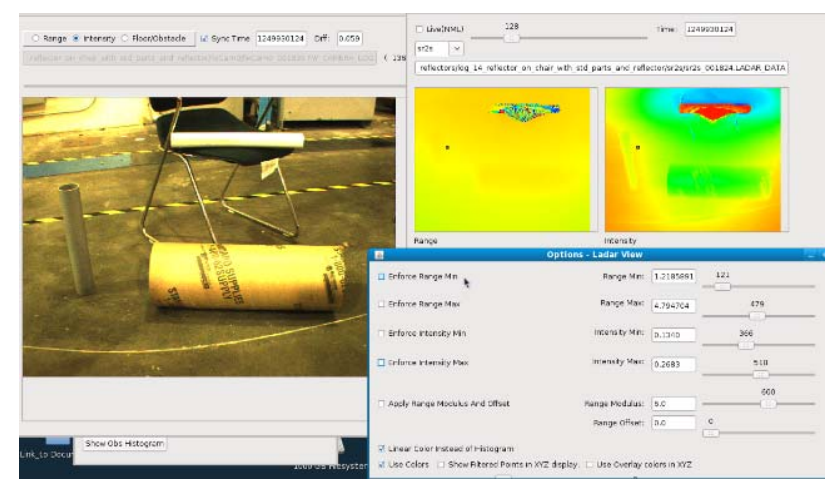

(b)

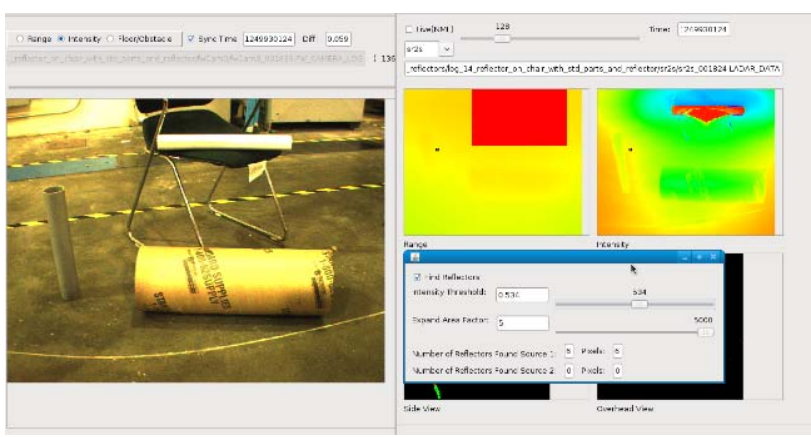

(c)

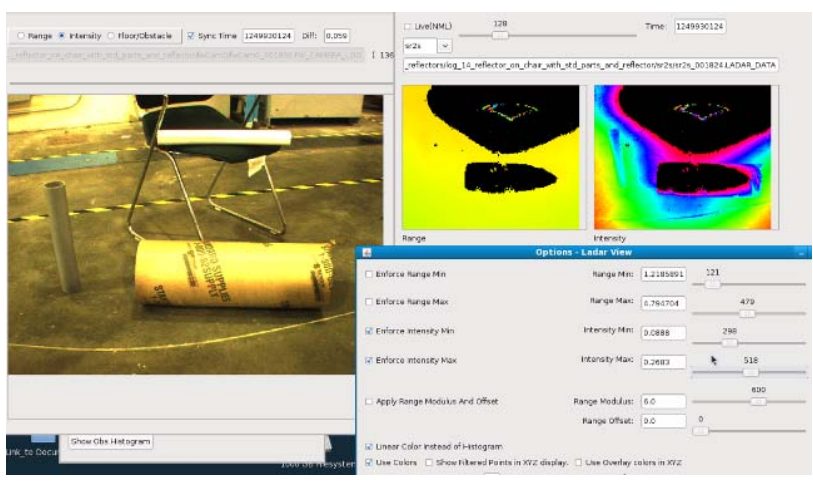

(d)

Figure 7: Color camera (left) and 3D Flash LIDAR intensity and range data (right) of (a) no reflector in the scene, (b) a reflector lying on the chair, (c) a reflector on the chair where the threshold algorithm has removed the highly reflective region from the data, (d) a reflector in the chair where the masking algorithm has removed the region of high reflectivity.

An engineering solution to the reflector problem would be to mount the sensor so that it is less likely to see the reflectors as shown in Figure 8. The floor could be flagged so that the sensor does not detect it as an obstacle, given the known sensor height. Unfortunately, this would leave the absolute positioning system's sensor above the field of view of the obstacle detection sensor, and thus unprotected by the sensor.

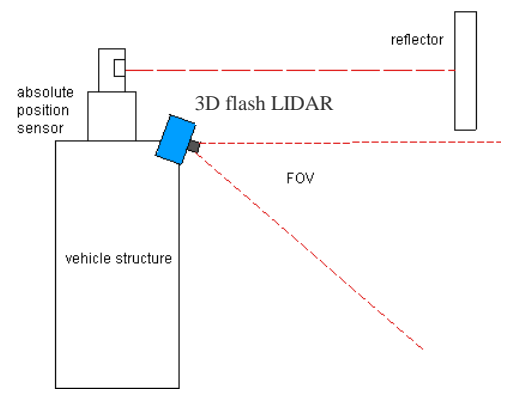

Figure 8: Drawing of a possible mounting scheme for the 3D Flash LIDAR sensor to not detect absolute positioning system reflectors.

The results of the reflectance experiments were that:

- Using sensor software drivers programmed to automatically threshold out highly reflective objects could improve bad range data issues. 
- Masking the upper sensor LED’s removed some image distortion. Better is to use a non-reflective surface just above the camera lens to block the upper LED's. And perhaps even better is not to use the data by masking it using software.

- Mounting the sensor specifically to detect obstacles well below (i.e., all data captured) or just below (i.e., Figure 5) the reflector heights could eliminate or greatly reduce position sensor reflector interference with the sensor.

For a second part of this experiment, we used a second 3D Flash LIDAR with a similar light source and pointed it directly at the first LIDAR. This experiment provided information about how well the sensor would function when a passing vehicle had similar sensors onboard. The results were that the sensor demonstrated no visually detectable change in range measurements when an LED array light source from a similar sensor passed by. This is probably due to the extreme unlikelihood that one sensor would send out its illumination flash at the same time as the other sensor was in receive mode.

\subsection{Detection of Forklift Tines}

As suggested by an AGV manufacturer, NIST recently measured forklift tines using the 3D Flash LIDAR. Full details of this experiment can be reviewed in [7]. The issue is that forklift tines and other obstacles can overhang the path of automated guided vehicles or other forklifts where 2D line scanning LIDAR may go undetected. We overlaid the images on an image from a color camera to provide a clear view of the tines or other obstacles detected. All measurements were taken dynamically while moving the sensor towards the forklift tines.

The 3D Flash LIDAR sensor and a color camera were mounted together with the camera lens just behind the flash sensor (see Figure 9. The camera FOV is slightly larger than that of the 3D Flash LIDAR. The two sensors were angled such that the 3D Flash LIDAR sensor detected the floor at a maximum distance of $6 \mathrm{~m}$. This setting: allowed a known sensor-to-floor distance to be used in the data processing algorithm, eliminated the highly-reflective objects above the FOV, and eliminated the cluttered background.

The forklift tines were set at two different heights $0.25 \mathrm{~m}$ (10 in) and $0.5 \mathrm{~m}$ (20 in). No preparation of the tines (e.g., paint, sand, etc.) was done. The cart was pushed towards the forklift tines at approximately $0.09 \mathrm{~m} / \mathrm{sec}(0.3 \mathrm{ft} / \mathrm{sec})$ during most data captures and for one experiment the cart was pushed at $0.53 \mathrm{~m} / \mathrm{s}(1.75$ $\mathrm{ft} / \mathrm{sec}$ ). Figure 10 shows the range, intensity, and overlaid obstacle detection on a color image when viewing the forklift tines from the front and side.

After reviewing the results from the unprepared tines and floor experiments, the researchers decided to conduct three additional tests, including: paint the tines with fluorescent paint, cover the floor and combine the painted tines with the covered floor to see what improvements, if any, would result. For each of the additional tests, the above experimental procedure was repeated.

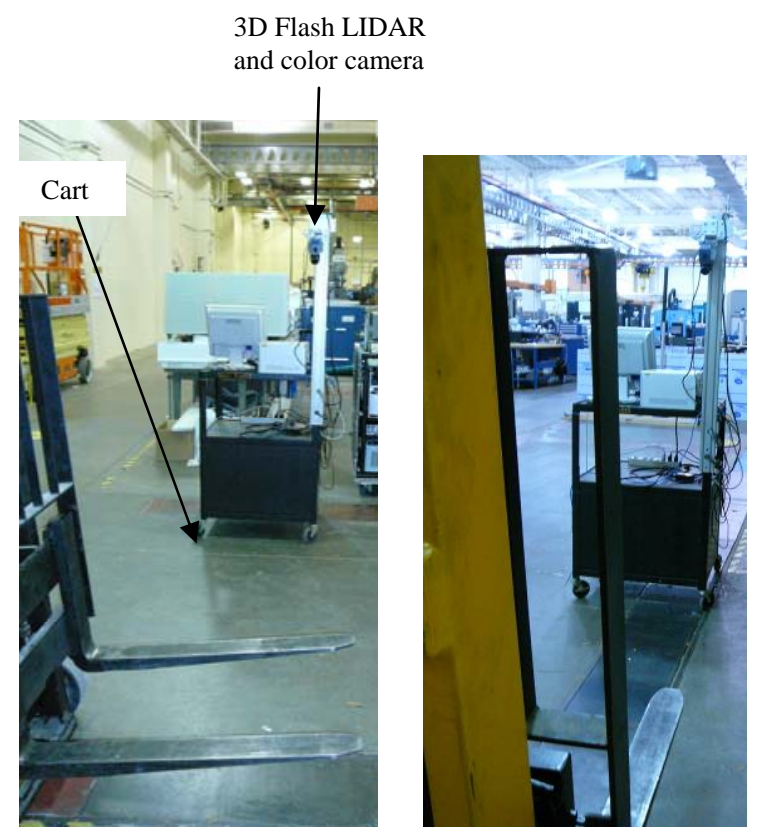

Figure 9: Experimental setup of a cart with 3D Flash LIDAR and color camera sensors (background) and raised forklift tines

(foreground). The forklift tines were measured from the side (left) and from the front (right).

Painted Tines: The tines were painted on only the edges perpendicular to the sensors for two reasons: 1) this surface is the smallest, yet facing surface to the sensor, and 2) this surface is the least likely to have paint removed when the tines are in use (i.e., wear against pallets is minimal for this surface). Only slight overspray covered the top surface of the tines.

Covered Floor: Another experiment included covering the floor with either white poster boards or with gray paint. This created a bright, uniform surface that was less detectable than the unprepared floor.

Combined Painted Tines with Covered Floor: The third experiment included both painted tines and covered floor. Figure 11 shows collected data from the 3D imager overlaid onto a photo of painted tines over painted floor where the floor in the foreground remains unpainted. As shown in the figure, there is little difference in this case between the painted and unpainted floors creating noise displayed as obstacles. However, the forklift and its tines were clearly detected as obstacles.

The interpretation of the data as good or bad was based substantially on subjective evaluation of image data from the sensor. Each data set (video) was reviewed and a human interpretation that the tines were detected required that a significant number of 3D Flash LIDAR pixels be clustered on the forklift tines. The percentage of time and the distances from the sensor at which the necessary pixel clustering on the tines appeared was noted. Table 1 shows the percentage detection at the full distance from the sensor start position to the tines, at 1 $\mathrm{m}$ and at $0.5 \mathrm{~m}$ for both front and side views of the tines. 

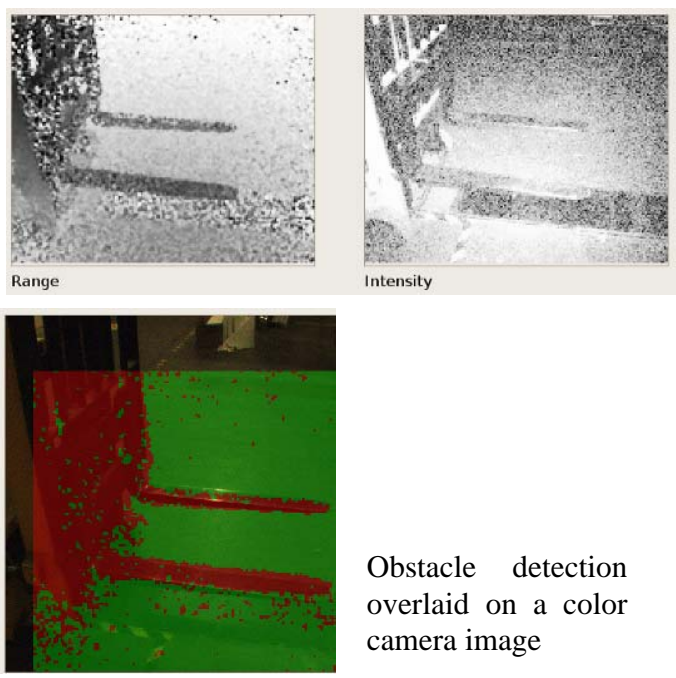

Intensity

Obstacle detection overlaid on a color camera image

(a)
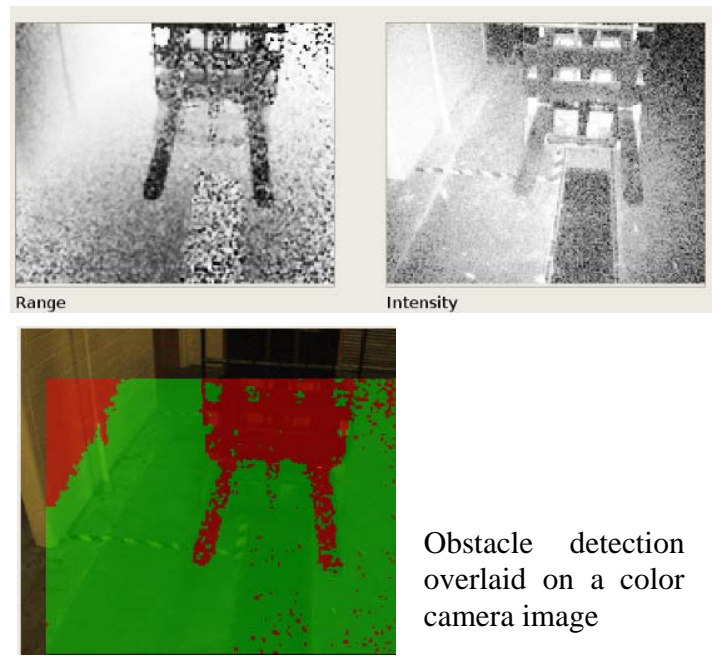

Obstacle detection overlaid on a color camera image

(b)

Figure 10: Data from the 3D Flash LIDAR showing range and intensity and obstacle detection overlaid on a color camera image of forklift tines as viewed from the (a) side and (b) front.

A key issue is that the 3D Flash LIDAR sensor processing program uses a height threshold to remove pixels beneath the forklift tines. Without this threshold, data from the floor and the tines may not appear different, and the tines would not be detected even by a human observer. Pixels with heights below 4 $\mathrm{cm}$ were removed, leading to two false negative results (Side 1, $4 \mathrm{~cm}$ and Front, $4 \mathrm{~cm}$ ). It may be preferable to develop an adaptive filtering algorithm that would allow the threshold to be lowered.

In some data sets, we saw 'bleeding' of obstacle detect data between, behind and in front of the tines. Figure 12 shows the tines being detected in the intensity and range images and also shows 'bleeding' of data perhaps from the left wall onto the floor in the bottom image. The painted tines joined with the floor covered with white poster boards where another unexpected phenomenon was detected as the front and rear tines were made into a large obstacle. Since the tines were clearly detected as shown in the intensity and range images, during our evaluation of fork tine detection we determined that this phenomenon did not change our forklift tines detection results. Therefore, our results show that during this test the 3D Flash LIDAR did detect the front tine.

Table 1: Percentage of Successful Tine Detection

\begin{tabular}{|l|c|c|c|}
\hline & \multicolumn{3}{|c|}{ Detect Percentage } \\
\hline $\begin{array}{c}\text { tine view (1 } \\
\text { from right, } \\
\text { = from left) } \\
\text { tine height } \\
\text { above the floor }\end{array}$ & $\begin{array}{c}\text { at the full } \\
\text { distance }\end{array}$ & $\begin{array}{c}\text { along the last 1 } \\
m\end{array}$ & $\begin{array}{c}\text { along the last } \\
0.5 \mathrm{~m}\end{array}$ \\
\hline Side 1, $4 \mathrm{~cm}$ & $0 \%$ & $0 \%$ & $0 \%$ \\
\hline Side $2,4 \mathrm{~cm}$ & $17 \%$ & $57 \%$ & $100 \%$ \\
\hline Front, $4 \mathrm{~cm}$ & $0 \%$ & $0 \%$ & $0 \%$ \\
\hline Side $1,8 \mathrm{~cm}$ & $50 \%$ & $100 \%$ & $100 \%$ \\
\hline Side $2,8 \mathrm{~cm}$ & $50 \%$ & $100 \%$ & $100 \%$ \\
\hline Front, $8 \mathrm{~cm}$ & $27 \%$ & $85 \%$ & $100 \%$ \\
\hline
\end{tabular}

A snapshot of data from the additional tests is shown in Figure 8 showing painted and unpainted floors and painted tines.

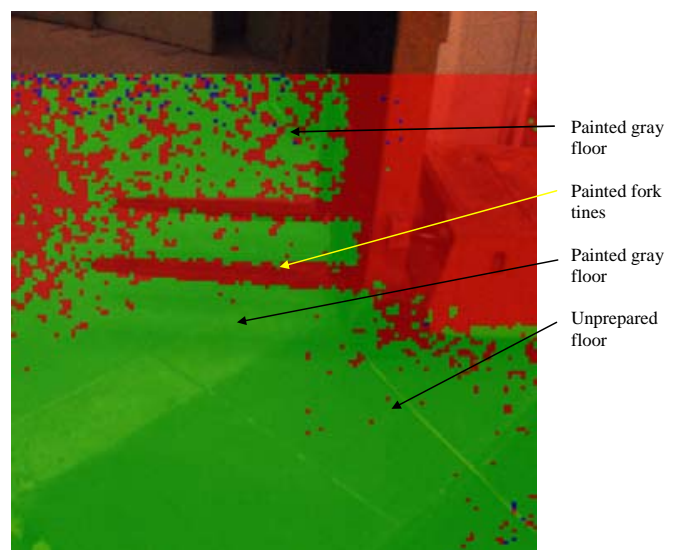

Figure 11: 3D Flash LIDAR sensor data overlaid onto a color camera image of painted forklift tines above a painted floor and beside unpainted floors.

We also showed that a 3D imager can be adjusted so as not to flag obstacles outside of a chosen area. This is useful for when the sensor is attached to a vehicle and the vehicle is driving along a narrow path and/or approaches a turn and the wall or obstacle in front of the vehicle prior to the turn is detected as an obstacle and in turn, stops or slows the vehicle. Figure 13 shows blue areas on the right and left sides of the vehicle path that have been excluded from processing. Although forklift tines appear in the image to be beyond the right edge (threshold) between light and dark colors, they are not. Some of the tines are shown as blue (grayscale black) and some are red (grayscale gray). The exclusion regions can be set for any side or range from the sensor and can be varied for complex paths and volumes if needed. Slow or stop regions can be set simultaneously in the same manner. 

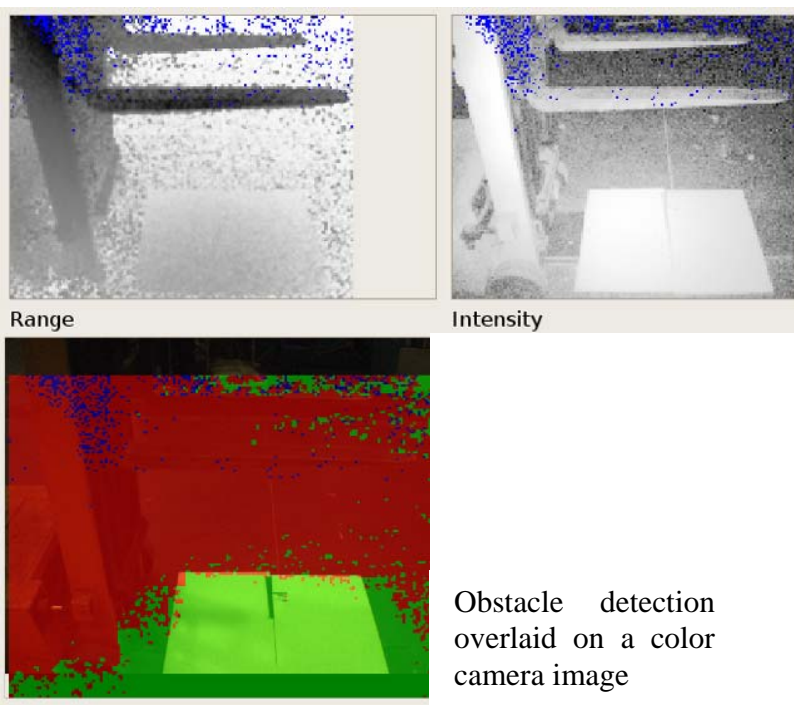

Intensity

Obstacle detection overlaid on a color camera image

Figure 12 - Range, intensity (top) and obstacle detection overlaid on a color camera image (bottom) of forklift tines. "Bleeding" detect data phenomenon is shown in the bottom image of detected forklift tines over a uniform poster-board floor covering.

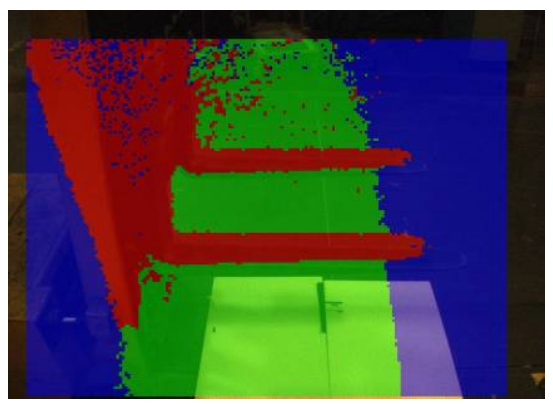

Figure 13 - Data from the 3D imager overlaid onto a color camera image after regions have been excluded from processing (blue areas) using an algorithm that flags when obstacles are outside the vehicle's path.

Results of the tine detection experiments, as detailed in [5], were as follows:

- Close range (last $1 \mathrm{~m}$ ) to the tines provides much more robust tine detection than longer ranges.

- The combination of sensors close to the tines and a high threshold height above the floor provides excellent tine detection.

- Higher tines are detected more often than lower tines due to the use of the height threshold.

- When the tines sides were painted with reflective yellow paint and the floor was covered with white poster board, the sensor performed very well.

- $\quad$ Slightly lower performance results were found when the floor was painted with light gray paint.

- Other color floor paints may provide similar results. However, high contrast between the tine and floor paint colors is expected to provide the best results.

\section{CONCLUSIONS}

The results of these experiments were used to enhance the ITSDF B56.5 standard to support new sensors such as advanced
3D imagers. In this paper, current and conceptualized ideal noncontact sensor configurations for manufacturing AGVs and forklift vehicles were presented. These concepts showed the need for performance measurements of advanced 3D imagers. NIST conducted experiments using a 3D Flash LIDAR sensor and a color camera on standard sized test pieces, coated and uncoated with materials and standard colors. The resulting measurements were used as background information to recommend changes to the ITSDF B56.5 standard with regard to non-contact sensors detecting standard test pieces. Before these experiments, two standard sized cylindrical test pieces were considered by the standard. Experimental results determined that a flat test piece should be added. We determined that the 3D sensor used is not a viable safety sensor for vehicles since: obstacles near highly reflective surfaces, returned skewed data, strange phenomenon of 'bleeding data' occurred, and very 'noisy' data returned when viewing non-uniform floor surfaces. Suggestions for sensor improvements, as we determined from our experiments, may be to support the sensor with robust data processing algorithms that detect highly reflective surfaces,and turn off or block sensor LED's. We found little effect from an external light source from another 3D imager on the sensor data. The percentage of tine detection shown in Table 1 provides a measure of the frequency with which the tines were detected. It shows that higher percentages of detection occur as range decreases. The snapshots and the percentage of detected tines data show that the 3D imager is not robust enough to detect black forklift tines $100 \%$ of the time and improves with painted tines with fluorescent paint above a uniform (painted) floor.

\section{REFERENCES}

[1] Occupational Safety and Health Administration website, http://www.osha.gov/SLTC/poweredindustrialtrucks/

[2] Industrial Truck Standards Development Foundation, (2005). ITSDF B56.5 Safety Standard for Guided Industrial Vehicles and Automated Functions of Manned Industrial Vehicles, http://www.itsdf.org.

[3] Mobility Open Architecture Simulation and Tools (MOAST) framework, https://sourceforge.net/projects/moast

[4] Roger Bostelman, Will Shackleford, Time of Flight Sensors Experiments Towards Vehicle Safety Standard Advancements, submitted to the Computer Vision and Image Understanding special issue on Time of Flight Sensors, April 2009.

[5] British Standard Safety of Industrial Trucks (1998). Driverless Trucks and their Systems. Technical Report BS EN 1525.

[6] Roger Bostelman, Will Shackleford, Test Report on Highly Reflective Objects Near the SR3000 Sensor, NIST Internal Report to Consortium CRADA Partners, February 27, 2008.

[7] Roger Bostelman, Will Shackleford, Test Report of Performance Measurements of a 3D Imager and Color Camera Viewing Forklift Tines, NIST Internal Report to Consortium CRADA Partners, September 24, 2008. 